\title{
El urbanismo y la arquitectura posmodernos, ¿capricho 0 necesidad?
}

\author{
Marissa Consiglieri Nieri de Chackal \\ Universidad Peruana de Ciencias Aplicadas \\ Recibido: 3 de julio del 2018 / Aprobado: 16 de agosto del 2018 \\ doi: 10.26439/limaq2019.n005.4527
}

\begin{abstract}
Aunque existen algunas características que distinguen al urbanismo y la arquitectura posmodernos, la corriente resulta dificil de comprender, cosa que, con frecuencia, incita el cuestionamiento de su validez. El presente artículo propone mostrar cómo y por qué la tendencia posmoderna, en la que se desenvuelven el urbanismo y la arquitectura actuales, representa parte importante de la solución a los problemas que aquejan el mundo, y es, por tanto, una necesidad. Esto se hará partiendo del análisis de dos textos fundamentales del movimiento y que dan paso a una nueva realidad arquitectónica: Complejidad y contradicción en la arquitectura, de Robert Venturi, y Construir, habitar, pensar, de Martín Heidegger.
\end{abstract}

posmodernidad, arquitectura, urbanismo, Venturi, Heidegger

\section{Postmodern urbanism and architecture, whim or need?}

Even though there are some characteristics that distinguish postmodern urbanism and architecture, the trend is difficult to understand and it often prompts questioning its validity. This paper aims to demonstrate how and why the postmodern tendency, where current urbanism and architecture develop, represents an important part of the solution to the problems that trouble the world and is therefore a need. For that purpose, two fundamental texts of the movement that lead out to a new architectural reality will be analyzed: Complexity and Contradiction in Architecture by Robert Venturi, and Building Dwelling Thinking by Martin Heidegger.

postmodernity, architecture, urbanism, Venturi, Heidegger 
Una arquitectura sustancial es más que un paraguas benevolente y protector; en su mejor expresión, interactúa con nosotros, en favor nuestro, informando nuestra memoria, haciéndonos más humanos. Es mucho más que un cobijo, un objeto de lucro, un estuche adecuado, un juguete caprichoso. Es el registro edificado de cómo hemos ordenado nuestros valores culturales, de qué y quiénes somos y de lo que creemos. (Roth, 2007, p. 612)

Sin lugar a ninguna duda, el registro actual deja mucho que desear. El estado del medioambiente a nivel mundial es preocupante. El agotamiento del planeta, el cambio climático, la crisis del agua, la pobreza, la injusticia y la violencia representan un peligro comprobado hoy en día. Su repercusión es el declive, a todo nivel, de la calidad de vida, no solo del ser humano, sino de todo ente viviente en la tierra. Es evidente que el problema no se enfrenta, ni ha sido enfrentado, de forma completa y apropiada ni por las organizaciones internacionales ni por los gobiernos de las grandes - y no tan grandes- potencias ni por los países llamados en desarrollo.

Este embrollo no ha sido causado únicamente por un desarrollo arquitectónico y urbano irresponsables, pero las deficiencias en el campo de la arquitectura no han atenuado la situación; la arquitectura, por tanto, ha sido, y es, parte del problema.

El presente artículo busca mostrar cómo y por qué el contexto posmoderno, en el que se desenvuelven el urbanismo y la arquitectura actuales, no es un hecho estético y autorreflexivo; por el contrario, tiene la posibilidad de ser parte integral del remedio a los problemas contemporáneos. El posmodernismo arquitectónico es, por tanto, una necesidad.

Para cumplir el propósito, se revisarán conceptos e ideas clave, en particular las de dos pensadores, Robert Venturi (Estados Unidos, 1925) y Martin Heidegger (Alemania, 1889-1976). De hecho, Venturi y Heidegger no son los únicos innovadores de su tiempo, pero una revisión completa de las teorías arquitectónicas iniciales está fuera del alcance del presente ensayo. Entonces, ¿por qué precisamente estos dos teóricos? Porque algunas de sus ideas engranan de modo tan justo que su exploración facilita la

1 Salvo que se especifique lo contrario, las traducciones han sido hechas por Marissa Consiglieri. 
comprensión y explicación de los fundamentos del posmodernismo, y en ellas se encuentran las raíces de esta nueva corriente que, a partir de los años 60 del siglo xx, modifica los paradigmas del planeamiento urbano y la arquitectura ofreciendo soluciones a muchas de las dificultades contemporáneas.

Primeramente, y antes de entrar de lleno en las propuestas de Venturi y Heidegger, es importante hablar del contexto en el que se da la tendencia posmoderna porque esto ayuda a conocer sus rasgos generales y a discernir su amplitud e impacto.

Jencks, por ejemplo, propone el posmodernismo como un amplio género inclusivo formado por un grupo de arquitectos que nace de la contracultura de los sesenta, pero que no solo incumbe a la arquitectura:

No solo Jane Jacobs, Robert Venturi y sus teorías de la complejidad, sino también Rachel Carson, el movimiento estudiantil, la sociedad postindustrial, la revolución electrónica, la contextualidad, el adhocismo, el metabolismo y más "ismos" de los que a uno le interese recordar. Es, entonces, un arcoíris que resiste los excesos del modernismo; una crítica, no un antimodernismo. (Jencks, 2002, p. 3)

El posmodernismo es, por ende, una tendencia diferente, diversa, compleja y contradictoria; una corriente que en muchos aspectos hace pisar tierra, sobre todo cuando se mira en oposición al modernismo. Al mismo tiempo, queda claro que, guste o no, en el ámbito posmoderno todo va.

Como toda corriente cultural importante, la posmodernidad tiene sus defensores y detractores; caso en punto, Bryan D. Palmer, quien advierte sobre el limitado futuro de la tendencia y se muestra contrario a ella. Él explica el pensamiento posestructuralista, y, por ende, el posmodernista como un fenómeno:

Muy difícil de precisar y definir con claridad porque celebra dispersión, diferencia y desestabilización: se desarrolla no como una teoría unificada, sino como conjuntos de círculos concéntricos en constante movimiento conectados en puntos congruentes, pero capaces de exigir, en cualquier momento, nuevos e inexplorados campos interpretativos. (Palmer, 1993, pp. 139-140) 
Efectivamente, no existe una teoría unificada, no hay ni manifiesto ni principios que permitan racionalizar y evaluar ordenada y metódicamente el posmodernismo, pero ese es el punto. Como bien explica Fernie: a posteriori, comprendemos que la posmodernidad nace como contracorriente de la modernidad; la esencia misma del fenómeno posmoderno lo define como rechazo a los principios del modernismo: el posmodernismo representa el "principio de los no principios y el repudio a la pureza de visión del modernismo" (1995, p. 351).

Best y Kellner examinan, acertadamente, el impacto específico de la corriente sobre la arquitectura. Ellos observan la transformación de la actitud formalista y pura moderna en una actitud liberal y juguetona caracterizada por un marcado eclecticismo. La quimera imaginada por Le Corbusier - $y$ arquitectos afines a él一, en la que gracias a la arquitectura se construye un mundo mejor, es aniquilada por "torres estériles y proyectos residenciales declarados en ruina" (Best y Kellner, 1991, p. 11). Simplemente dicho, la arquitectura moderna es autorreflexiva, narcisista y elitista. Su carácter hermético e introvertido no facilita la comunicación con el segmento más amplio de la población, con el usuario de a pie; por ende, tampoco responde a su contexto cultural.

Como respuesta, la propuesta posmoderna "censura la representación y el convencimiento de que la teoría refleja la realidad" (Best y Kellner, 1991, p. 4). En su oposición, el argumento posmoderno propone una actitud perspectivista y relativista frente a la teoría, y se afirma en la idea de que "[las] teorías, en el mejor de los casos, proveen una perspectiva parcial de sus objetos, y que todas las representaciones cognitivas del mundo son, históricas y lingüísticamente, [inter]mediadas" (Best y Kellner, 1991, p. 4). A su vez, la concepción posmoderna desestima las ideas de coherencia social y causalidad y propone las de multiplicidad, pluralidad, fragmentación e indeterminación. En fin, lo posmoderno rechaza los contenidos racionales $\mathrm{y}$ unificados intrínsecos del modernismo y promueve temas social y lingüísticamente descentralizados (Best y Kellner, 1991). Dicho de manera simple, la posmodernidad apuesta por una comunicación directa.

Es innegable el rol seminal de Robert Venturi en la búsqueda y el desarrollo del hecho arquitectónico — además de urbano- que comunique y responda 
directamente al usuario y su entorno.Venturi es el primer pensador y arquitecto en refutar la arquitectura prescriptiva y proscriptiva del modernismo, incluso antes de que el enfoque posmoderno haya sido identificado y su teoría haya sido desarrollada.

En Complexity and contradiction in architrecture (1992), Venturi propugna una nueva filosofia abriendo paso a la posibilidad de llevar a cabo una arquitectura diferente, que refleje, en las palabras de Roth ${ }^{2}$, nuevas aspiraciones y valores. Estos valores se evidencian en el texto de Venturi que citamos al final de este párrafo, que, además de ser un deleite, expresa, sin lugar a dudas, la esencia de la filosofía del arquitecto. Una filosofía que no solo expone su pensamiento sobre lo que debe ser, idealmente, la arquitectura, sino que revela un profundo conocimiento de la naturaleza humana. En otras palabras, Venturi no solo describe el edificio o la planta urbana ideal, él describe al ser humano, su pluralidad, su ambivalencia, su inconsistencia; es decir, al ser humano en toda su inherente complejidad y contradicción. Este reconocimiento implícito de la esencia humana señala un cambio sustancial: revela un interés profundo en el usuario que, intrínsecamente, resulta en la concepción de una arquitectura que propugna la mejora de su calidad de vida y entorno. Dice Venturi:

Prefiero los elementos híbridos a los "puros", los imprecisos a los "limpios", los distorsionados a los "rectos", los ambiguos a los "articulados", los tergiversados al igual que los impersonales, los aburridos que son a la vez "interesantes", los convencionales a los "diseñados", los complacientes a los excluyentes, los redundantes a los simples, los evocadores que son a la vez innovadores, los inconsistentes y los equívocos en lugar de los directos y "claros". Estoy en favor de una vitalidad confusa frente a una unidad evidente. Incluyo lo ilógico y proclamo la dualidad.

Defiendo la riqueza de contenido frente a la claridad del significado; la función implícita y la explicita. Prefiero "esto y lo otro" a "esto o aquello", "negro y blanco", y también gris, a blanco o negro. Una arquitectura de valor evoca muchos niveles de significado y combinaciones de perspectiva: su espacio y sus elementos devienen legibles y funcionales en diversos modos a la vez. (Venturi, 1992, p. 16)

2 Véase la primera cita. 
Como bien estima Stierli (2016), RobertVenturi reconoce la responsabilidad del arquitecto dentro del tejido social y sus palabras son "un fuerte llamado a la civilidad, y un llamado a una sociedad inclusiva en vez de una excluyente" (párr. 9).

De hecho, otros pensadores concuerdan con Stierli, y ninguno lo expresa mejor que Miguel Lacasta (2016) cuando dice lo siguiente:

Con este texto, se inaugura una nueva época en la arquitectura y el urbanismo. Se abren las puertas a una realidad operativa mucho más dinámica y compleja, abierta y empática, una arquitectura que jubila viejas reglas y abraza nuevos principios [cursivas de la autora]. (párr. 2)

Otro pensador importante para el desarrollo de la teoría arquitectónica contemporánea es Martin Heidegger ${ }^{3}$. En su transcendental ensayo de 1951, "Building, Dwelling, Thinking" ("Bauen Wohnen Denken") Heidegger analiza el acto de construir no como arte o técnica, sino como el dominio que constituye todo lo que es. El filósofo aspira a resolver dos preguntas: ¿qué es habitar? y ¿en qué medida construir pertenece a [es parte de] habitar? ("Building", 2006, p. 1)5.

Heidegger busca el nexo entre los términos construir, en el sentido de edificar, y habitar. A través del examen de la etimología de la palabra construir (bauen), advierte que ambos, construir (bauen) y habitar (wonhen), tienen la misma raíz. Asimismo, el escritor encuentra el vínculo entre construir/habitar y bin (ser). Heidegger explica, entonces, que ich bin (yo soy) y du bist (tú eres, segunda persona singular) significan también yo habito y tú habitas, respectivamente.Así, la locución habitar que expresa "la forma en que nosotros, los humanos, somos sobre la tierra es Buan, que significa 'habitar' ("Building”, 2006, p. 1). Por lo tanto, el ser humano es en tanto que habita. Es más, en el análisis de la palabra

3 El reconocimiento de la importancia de las ideas de Heidegger no representa la aprobación de su filiación al nazismo.

4 El ensayo fue presentado por primera vez en la Segunda Reunión de Darmastadt (Alemania) en 1951 y publicado en 1954.

5 El presente artículo no pretende ofrecer un análisis exhaustivo del ensayo de Heidegger; solo se presentan algunas ideas pertinentes simplificándolas y, al mismo tiempo, poniendo cuidado en no sacarlas de contexto. 
Bauen, Heidegger encuentra la asociación del término con vocablos como cuidar, proteger, cultivar, específicamente en el sentido de 'cultivar la tierra'. $\mathrm{Y}$ es en ese sentido de 'proteger' y 'cultivar' que podemos vincular el término, también, a la idea de producir: se cultiva lo que la tierra produce. Pero, al mismo tiempo, construir, en el sentido de levantar un edificio, es también habitar.Y es en este círculo perfecto de ideas — esta suerte de exégesis del términoque el autor regresa, hábilmente, al término construir. Construir, dice él, en el sentido de 'preservar' y 'cultivar' no significa manufacturar algo, "pero ambas formas de construir — construir/cultivar y construir/manufacturar - están estrechamente ligadas al concepto de habitar ("Building...", 2006, p. 1).

Heidegger enfatiza la importancia de tres ideas: "construir es realmente habitar; habitar es el modo en que los mortales somos en la tierra, y construir y habitar tienen dos acepciones: cultivar la tierra y alzar un edificio" ("Building", 2006, p. 2). En ellas está la clave: "el ser humano no habita porque ha construido, él construye para habitar porque, por naturaleza, los mortales son habitantes" [cursivas de la autora] ("Building", 2006, pp. 2-3).

Pero hay más por descubrir en la lengua. Según Heidegger, en el antiguo sajón existe la palabra wuon, que en el sajón gótico se convierte en wunian. Ambas palabras están estrechamente ligadas a bauen ('construir'), que, como ya se ha visto, significa también 'habitar'. El filósofo demuestra luego que hay un estado del ser comprendido en ambos vocablos: la expresión tiene un valor intrínseco que significa 'permanecer en paz', y el término paz (Friede) significa, a su vez, 'libre'. Este último se relaciona, de igual modo, a la locución das fry, que quiere decir 'preservar de daño o peligro'; es decir, ser protegido. Habitar, nos dice, significa 'estar [morar] en paz, establecerse en paz, dentro de la esfera libre que protege cada cosa [ente] en su naturaleza'. Como Sharr tan elegante y concisamente explica, "una edificación no debe ser comprendida como un objeto para ser admirado [...]. Más bien, es primordialmente parte de la experiencia humana continua de construir y habitar" (Sharr, 2007, p. 46). Es en este punto en el que las ideas de Venturi y Heidegger se encuentran ${ }^{6}$.

6 No es la intención sugerir que las ideas de Robert Venturi tienen su fuente en los escritos de Martin Heidegger; por otro lado, tampoco es descabellado proponer que el arquitecto haya conocido las ideas del filósofo, y que este último haya leído a Venturi. 
En una forma u otra, ambos consideran que hay una suerte de orden, o desorden, natural inherente al hecho arquitectónico y urbano que le permite cumplir con su rol de defensor, proveedor de refugio y protector de sus ocupantes — sean estos seres animados o inanimados-, al mismo tiempo que les otorga la libertad para desarrollarse en paz, porque cuando estas opciones están disponibles, "la arquitectura presenta y representa una cosmología y visión del mundo. Cuando estas cambian, todo el resto cambia" (Jencks, 2002, p. 3).

La posmodernidad crea opciones de cambio, y, aunque no sea a pasos agigantados, se avanza. Se avanza con estudios y teorías intertextuales en ciencia, ciencias sociales y humanidades que buscan mejorar la situación global.

En el campo de la arquitectura y el urbanismo per se, el mismo tipo de esfuerzo es representado por una corriente de pensamiento amplia e innovadora que, impulsada por las ideas de pensadores como Venturi y Heidegger, continúa alimentándose de sí misma, creciendo y evolucionando con el objetivo de satisfacer los requerimientos contemporáneos. Cambiando los paradigmas se acomodan, dentro de la complejidad y contradicción existentes, las necesidades, las exigencias y los derechos del ser humano y la sustentabilidad del entorno. Las consecuencias de lo contrario son conocidas: se crea un vórtice descendente, ese vórtice en el que se encuentra el mundo hoy.

En suma, la posmodernidad permite y conduce a un desarrollo urbano, suburbano y rural deliberado e inteligente, gracias a la evolución de una arquitectura de similar carácter. Asimismo, la tendencia posmoderna conecta la idea de permanecer y desarrollarse libremente y en paz con los conceptos de complejidad y contradicción, multiplicidad, pluralidad, diversidad, inclusión, etcétera. A esta concepción inicial se han venido agregando nociones como descentralización, contextualismo y sustentabilidad, que, a su vez, dan origen a diversas propuestas como la de la ciudad collage, los límites difusos, la planificación para la flexibilidad, el diseño ecológico,la configuración espacial orgánica, la renovada atención a la relación arquitectura/naturaleza, el jardín vertical, entre otros. Todas estas fórmulas son viables, inherentemente positivas y necesarias. Si se aplican con sensibilidad, imaginación y buen juicio, y se multiplican a lo largo y ancho del planeta, resultarán en una mejora sustantiva a la problemática global contemporánea. La postmodernidad, entonces, no es un capricho: es realmente una necesidad. 


\section{REFERENCIAS}

Best, S., y Kellner, D. (1991). Postmodern theory. Critical interrogations. Nueva York: Guilford Publications.

Building, dwelling, thinking by Martin Heidegger. (2006). En A. Hofstadter (trad.) [copia del texto publicado en Poetry, language, thought (1971), Nueva York: Harper Colophon Books]. Recuperado de http://faculty. arch.utah.edu/miller/4270heidegger.pdf

Fernie, E. (1995). Part III: Glosary of concepts. En Autor, Art history and its methods. A critical anthology (pp. 323-368). Londres: Phaidon Press Limited.

Jencks, C. (2002). The new paradigm in architecture: the language of postmodenism. Recuperado de https://books.google.ca/books?id=20bkr $\mathrm{u} 0 \mathrm{gzCMC} \&$ printsec $=$ frontcover $\& \mathrm{dq}=$ charles + jencks\&hl $=\mathrm{en} \& \mathrm{sa}=\mathrm{X} \&$ ved=0ahUKEwikjJedvbDaAhVrw4MKHQLtDvYQ6AEIKTAA\# $\mathrm{v}=$ onepage\&q\&f=true

Lacasa, M. (28 de marzo del 2016). Un suave manifiesto a favor de una arquitectura equívoca [publicación en un blog]. Recuperado de https:// axonometrica.wordpress.com/2016/03/28/un-suave-manifiesto-afavor-de-una-arquitectura-equivoca/

Palmer, B. D. (agosto de 1993). Critical theory, historical materialism, and the ostensible end of marxism: the poverty of theory revisited. International Review of Social History, 38(2), 133-162. Recuperado de https://www.jstor.org/stable/44582244

Roth, L. M. (2007). Understanding architecture, its elements, history, and meaning. Boulder: Westview Press.

Rowe, C., y Koetter, F. (1996). Collage city. En K. Nesbitt, Theorizing a new agenda for architecture. An anthology of architectural theory 1965-1995 (pp. 266-293). Nueva York: Princeton Architectural Press.

Sharr, A. (2007) Heidegger for architects. Londres: Routledge. 
Stierli, M. (22 de diciembre del 2016). Complexity and contradiction changed the way we look at, think and talk about architecture. The Architectural Review. Recuperado de https://www.architecturalreview.com/rethink/reviews/complexity-and-contradiction-changedhow-we-look-at-think-and-talk-about-architecture/10015872.article

Venturi, R. (1992). Complexity and contradiction in architrecture (2. $\left.{ }^{\mathrm{a}} \mathrm{ed}.\right)$ [copia en PDF]. Nueva York:The Museum of Modern Art, in association with the Graham Foundation for the Advanced Studies in the Fine Arts. 